Check for updates

Cite this: RSC Adv., 2017, 7, 49931

Received 22nd August 2017

Accepted 19th October 2017

DOI: $10.1039 / \mathrm{c} 7 \mathrm{ra09271g}$

rsc.li/rsc-advances

\section{Optically active blue-emitting carbon dots to specifically target the Golgi apparatus $\uparrow$}

\author{
Mengke Yuan, Yanjia Guo, Jianjia Wei, Jizhou Li, Tengfei Long and Zhongde Liu (DD* \\ Inspired by the knowledge that one usual method to impart chirality to metal nanoparticles is to passivate \\ them with ligands possessing chirality, in this context, a pair of optically active blue-emitting carbon dots \\ (CDs) were prepared using citric acid and D/L-penicillamine (D/L-Pen) as the carbon sources through \\ a two-step pyrolytic route. The second line of our research related to the effect of chiral CDs on \\ cytotoxicity reveals that CDs coated with D-Pen, show less cytotoxicity than L-Pen-coated CDs, although \\ generally the cytotoxicity of them is low. The subcellular localization examination reveals that the as- \\ prepared CDs could specifically target and directly illuminate the Golgi apparatus, in spite that no \\ obvious differences are observed between both of them. The discovery could provide a new alternative \\ avenue to trace the cell state by real-time monitoring the Golgi morphology.
}

\section{Introduction}

Chirality can be defined in such a way that the structure is nonsuperimposable with its mirror image, which is one of the key factors in molecular recognition, and has been considered to play an important role in many fields of analytical chemistry and biotechnology, especially in pharmaceutical science. Up to now although chirality at molecular level is exclusively understood, not much attention has been paid to the investigation of nanoscale chirality. The pioneering work by Schaaff and Whetten in 2000 was the first observation of the intense optical activity in giant gold-glutathione nanocluster compounds, ${ }^{1}$ which indicates that chiral effects are present in fact at the nanoscale. After that, efforts in the direction of creating inorganic chiral nanoobjects are largely directed towards combining the optical properties of chiral molecules with metal or semiconductor nanoparticles, especially thiolated chiral molecules capping nanoparticles. ${ }^{2-13}$ However, to the best of our knowledge, a very limited number of cases as to the efforts towards the development of chiral light emitting particles have been made ${ }^{\mathbf{1 4 - 1 7}}$ and therefore, how to obtain both highly luminescent and optically active particles nearly remains in burgeon stage and a worthwhile yet challenging undertaking, considering that chiral luminescent nanosized probes would provide very useful tools for both chemistry and biology.

As a category of chiral light emitting particles, optically active quantum dots (QDs), having been previously prepared using

Key Laboratory of Luminescent and Real-Time Analytical Chemistry (Southwest University), Ministry of Education, College of Pharmaceutical Sciences, Southwest University, Chongqing 400716, China. E-mail: lzdzhy@swu.edu.cn; Fax: +86-2368251048; Tel: $+86-23-68251910$

$\dagger$ Electronic supplementary information (ESI) available: Additional figures (Fig. S1-S8). See DOI: 10.1039/c7ra09271g chiral molecules as capping agents, ${ }^{\mathbf{1 4}, \mathbf{1 5}}$ are plagued with potential disadvantages related to environmental and biological toxicity when used on a large scale. Fluorescent carbon dots (CDs), since their first discovery in $2004,{ }^{18}$ being physicochemically and photochemically stable without photobleaching and rising as a star family in the field of biosensing and bioimaging, provide an environmentally benign alternative by virtue of their tunable biocompatibility, reactivity, ease of preparation and cost effectiveness. ${ }^{19}$ Generally, within the possible mechanisms that could explain the optical activity observed in chiral ligandprotected metal nanoclusters, it has been mentioned one that assumes an achiral core with chirality induced by chiral adsorption patterns, or with chiral ligands in achiral adsorption patterns. $^{20}$ Therefore, one usual method to impart chirality to metal nanoparticles is to passivate them with ligands possessing chirality. ${ }^{13}$ Inspired by the cases mentioned above, as a test case for chiral light emitting carbon nanoparticles (CNPs) synthesis, here the chiral photoluminescence (PL) CDs were attempted to synthesize using citric acid and $\mathrm{D} / \mathrm{L}$-penicillamine (D/L-Pen) as the carbon sources through a two-step pyrolytic route to bestow chirality on the CDs as shown in Scheme 1 . Compared with the recent works reported by Martin, in which the chiral graphene quantum dots (GODs) can be obtained by reaction of oxidized GODs with enantiomerically pure $(R)$ or $(S)$ 2-phenyl-1-propanol, ${ }^{21}$ the optically active surface passivation procedure described here provide an alternative avenue to obtain optically active light emitting CNPs.

Additionally, despite a significant surge in the number of investigations into the biological effects of different type, size and surface modification of nanoparticles, only limited knowledge is currently to elaborate the impact of nanoscale chirality on living organisms. ${ }^{22}$ Further, inspired by the knowledge that some biomolecules with abundant thiol groups such as 


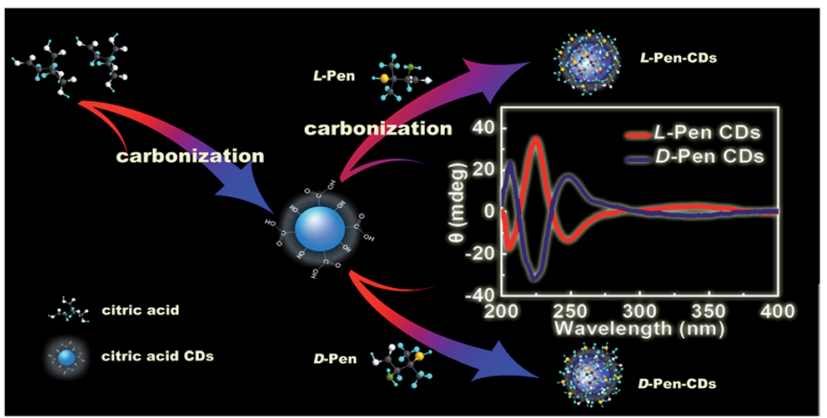

Scheme 1 Schematics of the formation of chiral light emitting CDs through a two-step pyrolytic route using citric acid and D/L-Pen as the carbon sources, respectively.

galactosyltransferase and protein kinase D are capable of anchoring in the Golgi apparatus, ${ }^{23,24}$ the second line of our research is focused on the effects of chiral PL CDs on cytotoxicity and subcellular localization. As a result, the pair of optically active CDs capped with different chiral forms of the Pen were found to show chirality-dependent cytotoxicity, with L-PenCDs being more cytotoxic than D-Pen-CDs. Impressively, subsequent subcellular localization examination revealed that the as-prepared CDs could specifically target and directly illuminate the Golgi apparatus due to the presence of abundant thiol functional groups, in spite that no sharp differences are observed between the L-Pen-CDs and D-Pen-CDs. Considering the superior photostability and long-term in situ imaging ability of the chiral light emitting CDs, the discovery could provide a better avenue to monitor the change of the Golgi apparatus at the single-cell level and may be also significant for the design of novel drug carriers for the Golgi-related diseases.

\section{Experimental}

\subsection{Materials}

L-Penicillamine (L-Pen), D-penicillamine (D-Pen) and L-alanine, Dalanine were purchased from Sigma-Aldrich (Shanghai, China). Citric acid and potassium hydroxide were commercially available from Aladdin Chemistry Co. Ltd (Shanghai, China). Mili-Q purified water (18.2 M $\Omega$ ) was used throughout the experiments. All the chemicals and solvents were of analytical grade and used as received without further purification.

\subsection{Characterization}

The optical properties were determined by an F-2500 spectrophotometer (Hitachi, Japan), a UV-3100 spectrophotometer (Hitachi, Japan), and a J-810 circular dichroism (CD) spectrometer (JASCO, Japan), respectively. The high-resolution transmission electron microscopy (HRTEM) images were taken with a Tecnai G2 F20 field emission transmission electron microscope (FEI, USA). The FT-IR spectrum of the as-prepared CDs was collected on a FTIR-8400S Fourier Transform Infrared spectrometer (Tokyo, Japan). The elemental composition and the valence information of the CDs were measured with an ESCALAB 250Xi X-ray photoelectron spectroscopy
(Thermo Scientific, America). The absolute quantum yield (QY) of the CDs was measured by an absolute PL QY spectrometer C11347 (Hamamatsu, Japan). Fluorescence imaging was conducted using a DSU live-cell confocal microscope (Olympus, Japan) system with laser excitations of CY3 and DAPI channel.

\subsection{Synthesis and purification of the optically active light emitting CDs}

The optically active light emitting CDs were prepared through a simple, low-cost and two-step pyrolytic process. Briefly, the citric acid was first added into a clear drying round-bottom flask, and then heated for $20 \mathrm{~min}$ in a thermostated oil bath at $200{ }^{\circ} \mathrm{C}$. After a slight cooling, $\mathrm{L}$-Pen (or D-Pen) was subsequently added and mixed uniformly with the former, and the mixture was heated for another $10 \mathrm{~min}$ at $150{ }^{\circ} \mathrm{C}$. Further procedures included neutralization with $\mathrm{KOH}$ solution, purification by filtering using $0.22 \mu \mathrm{m}$ membranes, and dialysis against deionized water for $48 \mathrm{~h}$. Finally, the products were obtained by a freeze-drying procedure. For comparison, the citric acid CDs and the alanine CDs were also prepared in a similar manner.

\subsection{Cell viability assays and PL imaging}

Human epithelial cells (Hep-2 cells), acquired from Cell Bank of Chinese Academy of Sciences (Shanghai, China), were added to each well of a 96-well plate $\left(1.0 \times 10^{5}\right.$ per $\mathrm{mL}, 100 \mu \mathrm{L}$ per well $)$. Plates were first maintained in an incubator $\left(37^{\circ} \mathrm{C}, 5 \% \mathrm{CO}_{2}\right)$ for $24 \mathrm{~h}$. Then the culture medium was replaced with $2 \%$ fetal bovine serum medium containing the obtained CDs with different concentration for another $24 \mathrm{~h}$. Finally, $10 \mu \mathrm{L}$ of Cell Counting Kit-8 (CCK-8) solution and $90 \mu \mathrm{L}$ medium were added into every cell well for $30 \mathrm{~min}$. The optical density (OD) of the mixture was measured at $450 \mathrm{~nm}$ with a Microplate Reader Model. The cell viability was estimated according to the following equation:

$$
\text { Cell viability }(\%)=\left(\mathrm{OD}_{\text {treated }}-\mathrm{OD}_{\mathrm{PBS}}\right) /\left(\mathrm{OD}_{\text {control }}-\mathrm{OD}_{\mathrm{PBS}}\right)
$$

$\mathrm{OD}_{\text {control }}$ was obtained in the absence of $\mathrm{CDs}$, $\mathrm{OD}_{\mathrm{PBS}}$ was the absorption value of $\mathrm{PBS}$, and $\mathrm{OD}_{\text {treated }}$ was obtained in the presence of CDs.

As for the PL cell imaging, the Hep-2 cells in RPMI 1640 supplemented with $2 \%$ fetal bovine serum were added to imaging dishes ( $1 \mathrm{~mL}$ per well). Then cells were cultured for $24 \mathrm{~h}$ at $37^{\circ} \mathrm{C}$ in a humidified $5 \% \mathrm{CO}_{2}$ atmosphere. After that, the culture medium was replaced with $1 \mathrm{~mL}$ RPMI 1640 containing $100 \mu \mathrm{g} \mathrm{mL} \mathrm{m}^{-1}$ of the $\mathrm{L}-\mathrm{CDs}$ and the $\mathrm{D}$-CDs, respectively, and incubated at the incubator for $2 \mathrm{~h}$. Further the cells were washed with PBS buffer for 3 times and fixed with Golgi-Tracker Red for 30 min at $4{ }^{\circ} \mathrm{C}$. Washing the cells with PBS buffer for 3 times again. The $100 \mu \mathrm{L} 2 \%$ fetal bovine serum was added into the imaging dishes for $30 \mathrm{~min}$ at $37^{\circ} \mathrm{C}$. After that the cells were washed with PBS and added $2 \%$ fetal bovine serum. Finally, the PL imaging photographs were captured with a DSU live-cell confocal microscope (Olympus, Japan) system at laser excitations of DAPI and CY3 channel. That is, the CDs was excited at 
360-370 $\mathrm{nm}$ and detected with a barrier filter BA $420-460 \mathrm{~nm}$, and the Golgi Tracker Red was excited at 530-550 nm and detected with a barrier filter BA 575-625 nm, respectively.

\section{Results and discussion}

The chiral light emitting CDs were synthesized through a twostep pyrolytic route, and typically the citric acid was first heated at $200{ }^{\circ} \mathrm{C}$ in a round flask for 20 min, during which primary CDs with weak emission were formed. After a slight cooling, L-Pen (or D-Pen) was subsequently added and mixed thoroughly. The mixture was heated at $150{ }^{\circ} \mathrm{C}$ for another $10 \mathrm{~min}$. During the synthetic process, the formed primary CDs, with a low absolute photoluminescence (PL) quantum yields (QYs) of $4.8 \%$, in practice acted as the carbon nucleus for the subsequent formation of chiral light emitting CDs. That is, after introduction of the L-Pen and D-Pen, respectively, the polymerization reactions, a second carbonization process, then occurred between the carbon nucleus and Pen enantiomers to form the optically active light emitting CDs, the absolute PL QYs rising to as high as $49.2 \%$ and $52.7 \%$, respectively.

The UV-vis absorption spectra display that the as-prepared LPen-CDs have characteristic absorption peak at $344 \mathrm{~nm}$, and concomitantly, a shoulder peak appears at about $247 \mathrm{~nm}$ as indicated by the arrow in Fig. 1a, the typical characteristic absorption spectra illustrating the formation of CDs. The strong absorption at $247 \mathrm{~nm}$ ascribes to a large amount of $\pi$-electrons in the $\mathrm{sp}^{2}$ hybridised islands, while the broad one at $344 \mathrm{~nm}$ is mainly attributed to the $\mathrm{n}-\pi *$ transition of the $\mathrm{C}=\mathrm{O}$ bond. ${ }^{25}$ Under the irradiation of $365 \mathrm{~nm}$ UV light lamp, the CDs aqueous solution present bright blue colour (the inset of Fig. 1a) and the maximum PL emission peak is located at $439 \mathrm{~nm}$ when excited at $363 \mathrm{~nm}$. Unlike most CDs, the emission wavelength of the Pen-CDs is independent of the excitation wavelength. When
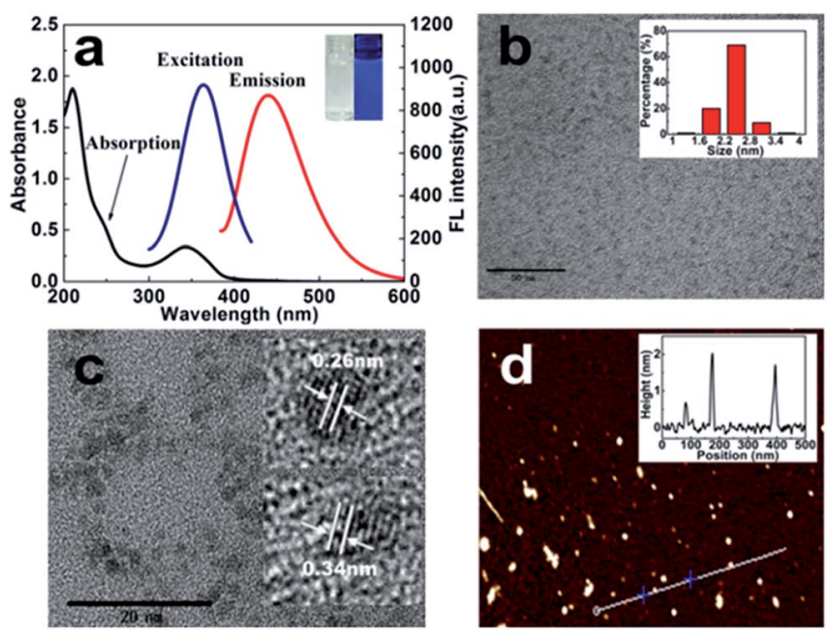

Fig. 1 Characterization of the as-prepared L-Pen-CDs. (a) Absorption and PL spectra of L-Pen-CDs, the inset showing photographs of LPen-CDs under daylight and 365 nm UV light; ( $b$ and c) TEM images of the L-Pen-CDs, the insets are size distribution and representative lattice fringes, respectively; (d) AFM image L-Pen-CDs, the inset showing the section analysis along the scored line. the excited light beam is changed from 300 to $390 \mathrm{~nm}$, the emission wavelength shows nearly no shift (Fig. S1†). Usually, excitation-dependent photoluminescence (PL) behaviors of CDs reflect effect from particles of different sizes in sample and a distribution of different surface states ${ }^{26,27}$ However, the size of the Pen-CDs is not very uniform, and actually ranges from 1.0 to $4.0 \mathrm{~nm}$ (the inset of Fig. 1b), which is calculated by measuring the average size of 100 single carbon nanodot (Nano Measure Software). Therefore, the excitation-independent PL behavior suggests that the PL properties of the Pen-CDs are depend on the surface states rather than the morphology, and the surface states of the Pen-CDs should be rather uniform. The high resolution TEM images (the inset of Fig. 1c) clearly show that the as-prepared CDs have the lattice spacing of $0.26 \mathrm{~nm}$ and $0.34 \mathrm{~nm}$, which may be attributed to the (100) facet of graphitic carbon and the spacing between graphene layers (002), respectively. ${ }^{28,29}$ The atomic force microscopy image of L-Pen-CDs reveals that their topographic heights are from 0.8 to $1.8 \mathrm{~nm}$ (Fig. 1d), indicating that the CDs consist primarily of about 2 to 4 layer of graphene-like sheets. If the D-Pen instead of the L-Pen is used in the second carbonization process, similar results can also be obtained for the D-Pen-CDs (Fig. S2 $\uparrow$ ).

Meaningfully, the ellipticity of the CDs was obtained by CD spectroscopy, showing that the chirality of the Pen precursors can be transferred to the CDs through optically active surface passivation procedure. In the $\mathrm{CD}$ spectra, an almost perfect mirror-image relationship was observed between the L-Pen-CDs and the D-Pen-CDs, and the measurable cotton effects were obviously exhibited at $\sim 206 \mathrm{~nm}, \sim 225 \mathrm{~nm}, \sim 247 \mathrm{~nm}$ and $\sim 337 \mathrm{~nm}$ (Fig. 2a), with the anisotropy factors (or $g$ factor, defined as $g=\Delta \varepsilon / \varepsilon$, where the intensity of the molar dichroic absorption $\Delta \varepsilon$ is normalized to the extinction coefficient $\varepsilon$ ) of $6.33 \times 10^{-4}, 1.86 \times 10^{-3}, 1.11 \times 10^{-3}$ and $2.73 \times 10^{-4}$, respectively (Fig. 2b), but no obvious $\mathrm{CD}$ response at all for the rac-Pen-CDs. Note that optically active Pen molecules contribute to the CD signal only in the deep UV region (Fig. S3, $\uparrow$ the ordinary absorption and CD spectra of Pen enantiomers), and the CD scans of as-prepared optically active CDs are strikingly different from those of the free L-Pen and D-Pen. Therefore, the structured CD spectra observed in the optically active CDs should be through different quantized transitions that resulted from the bandgap transitions from conjugated $\mathrm{sp}^{2}$-domanins. ${ }^{30}$
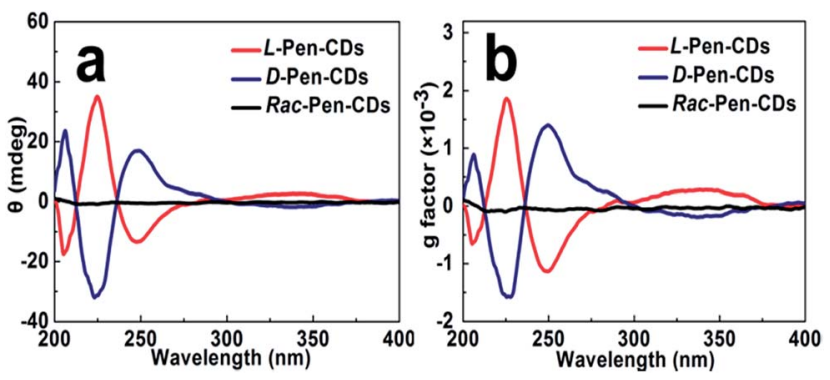

Fig. 2 The chiroptical behaviour of as-prepared light emitting CDs. (a) The CD spectra of L-Pen-CDs, D-Pen-CDs and rac-Pen-CDs; (b) the corresponding anisotropy factors ( $g$ factors) for all of them, respectively. 
It should be noted that the conditions of the pyrolytic process were critical to the formation of the chiral light emitting CDs. For example, after adding the Pen enantiomers into the formed primary CDs, we optimized the second pyrolytic reaction time, and it was found that the carbonization reaction at $150{ }^{\circ} \mathrm{C}$ for 10 min between the carbon nucleus and Pen enantiomers could result in the largest optically active CDs. By further prolonging the time of reaction, the CD signals of both $\mathrm{L}^{-}$ Pen-CDs and D-Pen-CDs at $247 \mathrm{~nm}$ gradually decrease (Fig. S4 $\uparrow$ ), illustrating that the optically active light emitting CDs have been formed in the initial stage of the reaction and the sustained high temperature reaction could exert a detrimental effect on the large chiroptical activity of the obtained CDs. In addition, the adopted pyrolytic temperature of $150{ }^{\circ} \mathrm{C}$, much lower than the melting temperature of Pen at $206^{\circ} \mathrm{C}$ is critical to the formation of the functional chiral PL CDs, also highlighting the impact of temperature on the chiroptical activity of CDs.

A theoretical study that describes the dissymmetrical field model demonstrates that the induced optical activity in chiral monolayer protected metal clusters could arise from achiral cores perturbed by a chiral field originating from the adsorbates. ${ }^{20}$ Therefore, as for the mechanism that chirality is bestowed on the CDs through optically active surface passivation, the plausible explanation is that a vicinal effect, which is caused by an achiral core with chiral ligands in achiral adsorption patterns, should be responsible for the origin of nanoscale chirality. In other words, chiral Pen adsorbates induce a chiral perturbation on the carbon core making it optically active. The results that the primary carbon nucleus, only resulted from the carbonization of citric acid, is achiral (Fig. S5 $\dagger$ ), and the chiral PL CDs are obtained after a second carbonization process occurred between the carbon nucleus and Pen enantiomers, also support the contention mentioned above. If the achiral rac-Pen instead of L-Pen or D-Pen is used in the second carbonization process, there is no obvious CD response at all for the rac-Pen-CDs (Fig. 2) due to the net optical activity elimination, indicated that only chiral adsorbates could act as a perturbing electrostatic field to break down the symmetry of the electronic states in the CDs.

FTIR spectra (Fig. 3a) exhibits characteristic absorption band of S-H stretching vibrations at $2545 \mathrm{~cm}^{-1}$, and stretching vibration peaks at $1604 \mathrm{~cm}^{-1}$ and $1189 \mathrm{~cm}^{-1}$ indicate the existence of $\mathrm{C}=\mathrm{O}, \mathrm{C}-\mathrm{N}-\mathrm{C}$ groups in the obtained $\mathrm{L}-\mathrm{Pen}-\mathrm{CDs}$. The $\mathrm{X}-$ ray photoelectron spectroscopy (XPS) wide-scan of them (Fig. 3b) shows four peaks at 530.9, 376.7, 285.4 and $164.1 \mathrm{eV}$, corresponding to $\mathrm{O} 1 \mathrm{~s}, \mathrm{~N} 1 \mathrm{~s}, \mathrm{C} 1 \mathrm{~s}$ and $\mathrm{S} 2 \mathrm{p}$, respectively, obviously reveal that the as-prepared CDs are doped with $\mathrm{N}$ and $\mathrm{S}$ atoms. The high-resolution XPS spectrum of C 1s (Fig. 3c) reveals the presence of various carbon bonded species such as $\mathrm{C}-\mathrm{C}(284.5 \mathrm{eV}), \mathrm{C}-\mathrm{S}(285.4 \mathrm{eV})$ and $\mathrm{C}=\mathrm{O}(288.0 \mathrm{eV})$. The $\mathrm{S} 2 \mathrm{p}$ spectrum (Fig. 3d) demonstrates that the peaks at 163.2 and $163.8 \mathrm{eV}$ are corresponding to the binding energy for the $\mathrm{C}-\mathrm{S}-\mathrm{H}$ units. ${ }^{28}$ The peaks at $530.7 \mathrm{eV}$ and $531.4 \mathrm{eV}$ owe to $\mathrm{C}=\mathrm{O}$ and $\mathrm{C}-$ $\mathrm{O}-\mathrm{C} / \mathrm{C}-\mathrm{OH}$, respectively ${ }^{31}$ (Fig. 3e). The peak at $399.8 \mathrm{eV}$ in the $\mathrm{N}$ 1s spectrum (Fig. 3f) demonstrates the presence of $\mathrm{C}-\mathrm{N}-\mathrm{C}$ bonds. ${ }^{32}$ Similar results also occur to the D-Pen-CDs (Fig. S6 $\dagger$ ). Hence, from both the XPS and FTIR measurements we have
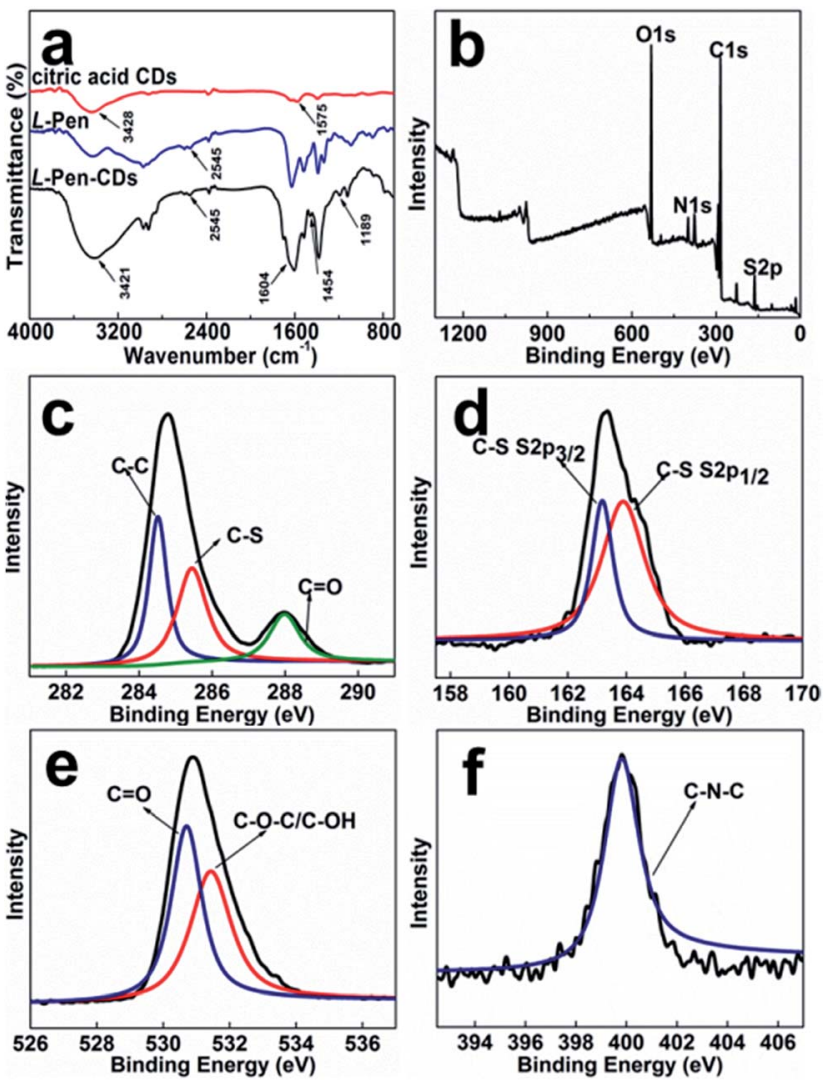

Fig. 3 FT-IR spectra of the critic acid CDs, the L-Pen alone and the LPen-CDs (a); XPS survey scan of the L-Pen-CDs (b); high resolution XPS spectrum of $C 1 s(c), S 2 p(d), O 1 s(e)$, and $N$ 1s (f) for the L-Pen-CDs.

demonstrated that the surfaces of as-prepared CDs are mainly full of thiol and hydroxyl groups. From this we can speculate that through the formation of amide bonds in the second carbonization process, the optically active Pen molecules could be attached to the surface of primary CDs, also supporting our

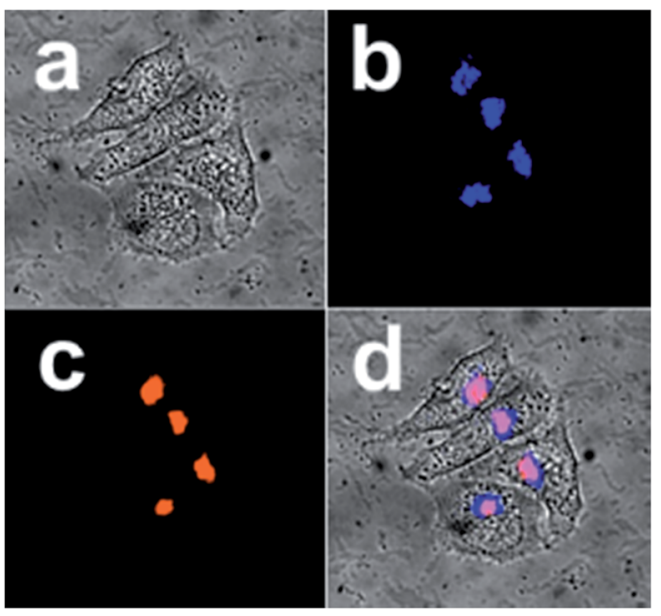

Fig. 4 Confocal microscopy images of Hep-2 cells treated with the DPen-CDs and the Golgi-Tracker Red. (a) Bright field images; (b) DAPI channel; (c) CY3 channel; (d) merged fluorescence and bright field images. 
claims that a vicinal effect should be responsible for the observed optically active light emitting CDs.

The as-prepared CDs are photostable even illuminated under the light of $150 \mathrm{~W}$ Xe lamp for $30 \mathrm{~min}$ (Fig. S7a $\dagger$ ), and are also very stable in salt solution even if the concentrations of $\mathrm{NaCl}$ is as high as 4.0 M (Fig. S7b $\dagger$ ), indicating that they would not be affected in the similar ion-rich biological environments. Moreover, the PL intensity of the CDs gets slightly increased in the $\mathrm{pH}$ range from 2.2 to 5.0, and scarcely changed in the $\mathrm{pH}$ range from 5.0 to 12.0 (Fig. S7c $\dagger$ ). In addition, we examine the antioxidant capacity of the pair of CDs enantiomers, and it is found that both the L-Pen-CDs and D-Pen-CDs are very stable and the PL intensity of them gets slightly decreased even if the concentration of the $\mathrm{H}_{2} \mathrm{O}_{2}$ reach $0.5 \mathrm{M}$ (Fig. S7d $\dagger$ ). The CKK-8 assay assessments show that the obtained CDs enantiomers impose dose-dependent toxicity to the cells, however, the cytotoxicity of CDs is generally very low, and the viability of Hep-2 cells remain greater than $80 \%$ even if incubated with an relatively high concentrations $\left(1.0 \mathrm{mg} \mathrm{mL}^{-1}\right)$. In addition, it is found that the CDs capped with different chiral forms of the Pen show chirality-dependent cytotoxicity, with L-Pen-CDs being more cytotoxic than $\mathrm{D}$-Pen-CDs for a given concentration (Fig. S8†), which provides important insight for designing more "smart" surface coatings and may open a new avenue for further development of CDs for biomedical imaging.

Further, in order to know whether the differences in subcellular localization exist between the present L-Pen-CDs and $\mathrm{D}$-Pen-CDs, the Hep-2 cells are exposed to the same concentrations of the D-Pen-CDs and L-Pen-CDs and the cell imaging is followed. Impressively, thanks to the remarkable cellular uptake and the high PL quantum yield, as shown in Fig. 4, it is found that the as-prepared CDs can specifically target the Golgi apparatus. Co-staining Hep-2 cells using the D-PenCDs and the Golgi-Tracker Red, a Golgi specific fluorescence dye, to confirm their subcellular location, show that the colocalised images of D-Pen-CDs match very well with those of Golgi-Tracker Red. The Pearson's correlation factor, used for assessing the co-localization efficiency, was higher than 0.84, indicating the preferential accumulation of the CDs in the Golgi apparatus. The galactosyltransferase and the protein kinase $\mathrm{D}$, with abundant thiol groups, are capable of anchoring in the Golgi apparatus, ${ }^{23,24}$ and therefore the precise Golgi localizing ability of the CDs could be largely ascribed to the thiol groups on the surface of them. If the Hep-2 cells were incubated with the free D-Pen and L-Pen molecules before the addition of the corresponding D-Pen-CDs and L-Pen-CDs, the Golgi targeting ability of D-Pen-CDs and L-Pen-CDs can be inhibited (Fig. S9†), with the Pearson's correlation factor of 0.31 and 0.37 , respectively. Further, the carbon dots prepared from precursors carrying no free thiol groups such as D-alanine and L-alanine, are found to have no Golgi targeting ability and to randomly spread in cells (Fig. S10†). These observations reinforce the existence of thiol groups on the surface of the as-prepared CDs to be necessary for targeting the Golgi apparatus.

But contrary to our expectation, no sharp differences in subcellular localization are observed between the D-Pen-CDs and L-Pen-CDs, and the latter can also specifically target the
Golgi apparatus (Fig. S11 $\dagger$ ), with the Pearson's correlation factor of 0.71 . Furthermore, the rac-Pen-CDs also show the targeting ability of the Golgi apparatus to some extent (Fig. S12 $\dagger$ ), however, compared with the D-Pen-CDs, it is very low and the Pearson's correlation factors is only 0.43, indicating that the different configurational CDs exert influence on the targeting ability of them towards the Golgi.

\section{Conclusions}

In summary, inspired by the knowledge that the synthesis of nanoparticles involved in optically active molecules easily induces unique electronic and chiroptical response of nanostructures, we present a two-step pyrolytic route using citric acid and $\mathrm{D} / \mathrm{L}-\mathrm{Pen}$ as the carbon sources, respectively, to obtain optically active light emitting CDs. The vicinal effect, one of the dissymmetric field models that have been formulated to explain the nanoscale chirality on the nanostructures, is proposed to elucidate the origin of the chiroptical activity. Our work on the effect of chiral PL CDs on cytotoxicity reveal that CDs coated with D-Pen, being pharmaceutically and clinically useful, show less cytotoxicity than L-Pen-coated CDs, although the cytotoxicity of them is low. In addition, the as-prepared CDs could specifically target and directly illuminate the Golgi apparatus, in spite that no obvious differences are observed between both of them. The discovery could provide a new avenue to trace the cell state by real-time monitoring the morphology of the Golgi, and may be also significant for the design of new drug carriers for Golgi-related diseases.

\section{Conflicts of interest}

There are no conflicts of interest to declare.

\section{Acknowledgements}

This work was financially supported by the National Natural Science Foundation of China (21205096) and the Fundamental Research for the Central Universities (XDJK2014B023).

\section{Notes and references}

1 T. G. Schaaff and R. L. Whetten, J. Phys. Chem. B, 2000, 104, 2630-2641.

2 H. Yao, K. Miki, N. Nishida, A. Sasaki and K. Kimura, J. Am. Chem. Soc., 2005, 127, 15536-15543.

3 N. Nishida, H. Yao, T. Ueda, A. Sasaki and K. Kimura, Chem. Mater., 2007, 19, 2831-2841.

4 Q. Xu, S. Kumar, S. Jin, H. Qian, M. Zhu and R. Jin, Small, 2014, 10, 1008-1014.

5 O. Lopez-Acevedo, H. Tsunoyama, T. Tsukuda, H. Hakkinen and C. M. Aikens, J. Am. Chem. Soc., 2010, 132, 8210-8218.

6 A. Sanchez-Castillo, C. Noguez and L. L. Garzon, J. Am. Chem. Soc., 2010, 132, 1504-1505.

7 N. Cathcart and V. Kitaev, J. Phys. Chem. C, 2010, 114, 1601016017. 
8 H. Qian, M. Zhu, C. Gayathri, R. R. Gil and R. Jin, ACS Nano, 2011, 5, 8935-8942.

9 X. Yang, L. Gan, L. Han, D. Li, J. Wang and E. Wang, Chem. Commun., 2013, 49, 2302-2304.

10 M. Farrag, M. Tschurl and U. Heiz, Chem. Mater., 2013, 25, 862-870.

11 C. Zeng, T. Li, A. Das, N. L. Rosi and R. Jin, J. Am. Chem. Soc., 2013, 135, 10011-10013.

12 S. Knoppe and T. Burgi, Acc. Chem. Res., 2014, 47, 1318-1326.

13 R. Kobayashi, Y. Nonoguchi, A. Sasaki and H. Yao, J. Phys. Chem. C, 2014, 118, 15506-15515.

14 M. P. Moloney, Y. K. Gun'ko and J. M. Kelly, Chem. Commun., 2007, 38, 3900-3902.

15 T. Nakashima, Y. Kobayashi and T. Kawai, J. Am. Chem. Soc., 2009, 131, 10342-10343.

16 Y. L. Zhou, M. Yang, K. Sun, Z. Y. Tang and N. A. Kotov, J. Am. Chem. Soc., 2010, 132, 6006-6013.

17 R. S. Li, P. F. Gao, H. Z. Zhang, L. L. Zheng, C. M. Li, J. Wang, Y. F. Li, F. Liu, N. Li and C. Z. Huang, Chem. Sci., 2017, 8, 6829-6835.

18 X. Xu, R. Ray, Y. Gu, H. J. Ploehn, L. Gearheart, K. Raker and W. A. Scrivens, J. Am. Chem. Soc., 2004, 126, 12736-12737.

19 D. Iannazzo, I. Ziccarelli and A. Pistone, J. Mater. Chem. B, 2017, 5, 6471-6489.

20 C. Noguez and I. L. Garzon, Chem. Soc. Rev., 2009, 38, 757771.

21 M. V. Nakagawa, L. R. Perez, M. A. Herranz and N. Martin, Chem. Commun., 2016, 52, 665-668.
22 Y. Y. Li, Y. L. Zhou, H. Y. Wang, S. Perrett, Y. L. Zhao, Z. Y. Tang and G. J. Nie, Angew. Chem., Int. Ed., 2011, 50, 5860-5864.

23 D. Aoki, Proc. Natl. Acad. Sci. U. S. A., 1992, 89, 4319-4323.

24 Y. Maeda, G. V. Beznoussenko, J. Van Lint, A. A. Mironov and V. Malhotra, EMBO J., 2001, 20, 5982-5990.

25 H. Zhu, X. Wang, Y. Li, Z. Wang, F. Yang and X. Yang, Chem. Commun., 2009, 34, 5118-5120.

26 Y. P. Sun, B. Zhou, Y. Lin, W. Wang, K. A. S. Fernando, P. Pathak, M. J. Meziani, B. A. Harruff, X. Wang, H. Wang, P. G. Luo, H. Yang, M. E. Kose, B. Chen, L. M. Veca and S. Y. Xie, J. Am. Chem. Soc., 2006, 128, 7756-7757.

27 Y. Q. Dong, H. C. Pang, H. B. Yang, C. X. Guo, J. W. Shao, Y. W. Chi, C. M. Li and T. Yu, Angew. Chem., Int. Ed., 2013, 52, 7800-7804.

28 Y. Dong, H. Pang, H. B. Yang, C. Guo, J. Shao, Y. Chi, C. M. Li and T. Yu, Angew. Chem., Int. Ed., 2013, 52, 7800-7804.

29 J. Ge, M. Lan, B. Zhou, W. Liu, L. Guo and H. Wang, Nat. Commun., 2014, 5, 4596.

30 M. R. Goldsmith, C. B. George, G. Zuber, R. Naaman, D. H. Waldeck, P. Wipf and D. N. Beratam, Phys. Chem. Chem. Phys., 2006, 8, 63-67.

31 Q. Xu, P. Pu, J. Zhao, C. Dong, C. Gao, Y. Chen, J. Chen, Y. Liu and H. Zhou, J. Mater. Chem. A, 2015, 3, 542-546.

32 B. B. Chen, H. Liu, C. Z. Huang, J. Ling and J. Wang, New J. Chem., 2015, 39, 1295-1300. 